\section{Dwarf Apple Germplasm Release US B1, US B2, and US B3}

\author{
Miklos Faust' and Howard J. Brooks ${ }^{2}$ \\ Agricultural Research Service, Beltsville, MD 20705
}

Additional index words. Malus domestics, internode length, dormancy

Three dwarf apple (Malus domestica Borkh.) germplasm selections, US B1, US B2, and US B3, were released in July 1993 by the Agricultural Research Service, U.S. Dept. of Agriculture. The selections are available to researchers interested in short-internode genetic material. The three selections represent three sizes; US B1 is the smallest and US B3 is the largest. The three trees represent $\approx 70 \%$, $50 \%$, and $30 \%$ reduction over normal tree height.

\section{Origin}

The three germplasm selections resulted from open-pollinated sibcrossing of four selections of an original cross between two apple cultivara: 'Goldspur' × 'Redspur Delicious'. The original cross was made in 1965. Four selections were grown in a location $1.7 \mathrm{~km}$ away from other apple trees. Trees were allowed to intercrops naturally and 2000 of the resulting seeds were germinated and planted in a field in 1974. The 1974 population expressed a continuous segregation pattern for tree vigor and presence of spurs (Zagaja and Faust, 1983). From this population, three dwarf trees with different growth characteristics were selected and tested, and they are being released. Our trees were propagated by tissue culture from the original seedlings in 1984 and have been in the field for 9 years. Trees were not pruned, and the original tree habit is shown in Figs. 1 and 2.

\section{Description}

US B 1 is the most dwarf of the three selections (Fig. 1a). Original selection number was B 1 , row 5 tree 13 . Tree growth was tested on trees propagated by tissue culture on their own roots. Mean tree height at 9 years was 1.5 $\mathrm{m}$. Internode length of this selection is short compared to normal trees, but it changes during the season. Mean internode length is between 10 and $12 \mathrm{~mm}$ at the beginning of spring growth until the beginning of June when it shortens to $<5 \mathrm{mtn}$. Internode length responds to temperature. When temperatures reached $30 \mathrm{C}$ for $2-3 \mathrm{~h}$, internode length shortened

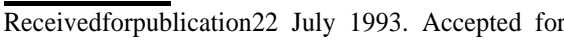
publication1 Dec. 1993. The cost of publishing this paper was defrayed in part by the payment of page charges.Under postal regulations, this paper thereforemustbeherebymarked advertisement solely to indicatethis fact.

${ }^{1}$ Fruit Laboratory.

${ }^{2}$ National Program Staff.
(Faust and Steffens, 1993). This is a reproducible characteristic under controlled conditions (Steffens, 1993). When internodes shorten, leaf area usually decreases also. After 1 June, leaves become narrow and appear not to be extended fully. This selection has no summer dormancy. Although other apple trees form terminal buds after producing $\approx 20$ to 25 leaves, US B1 continues to grow and produces $\geq 50$ leaves until late fall. US B 1 fruit look like that of 'Golden Delicious'. Fruit quality is close but not quite as good as that of 'Golden Delicious'. The fruit russetted under Beltsville, $\mathrm{Md}$, conditions. Tree branches are brittle because cells of wood and bark are smaller than those of the normal trees (Jaumien and Faust, 1984). Gibberellin content of this tree is genetically altered (Grochowska et al., 1984). After June, $\mathrm{GA}_{19}$ accumulates; at that time, there seems to be a block in the GA metabolism (Steffens and Hedden, 1992a). Hightemperature exposure for $2 \mathrm{~h}$ daily under controlled conditions duplicated the accumulation of $\mathrm{GA}_{19}$ that was observed in natural conditions (Steffens and Hedden, 1992b). To our knowledge, this tree represents the shortest internode-length dwarf tree reported in apples.

US B2 is also a dwarf tree (Fig. lb); however, it is larger than US B1. Main height of 9-

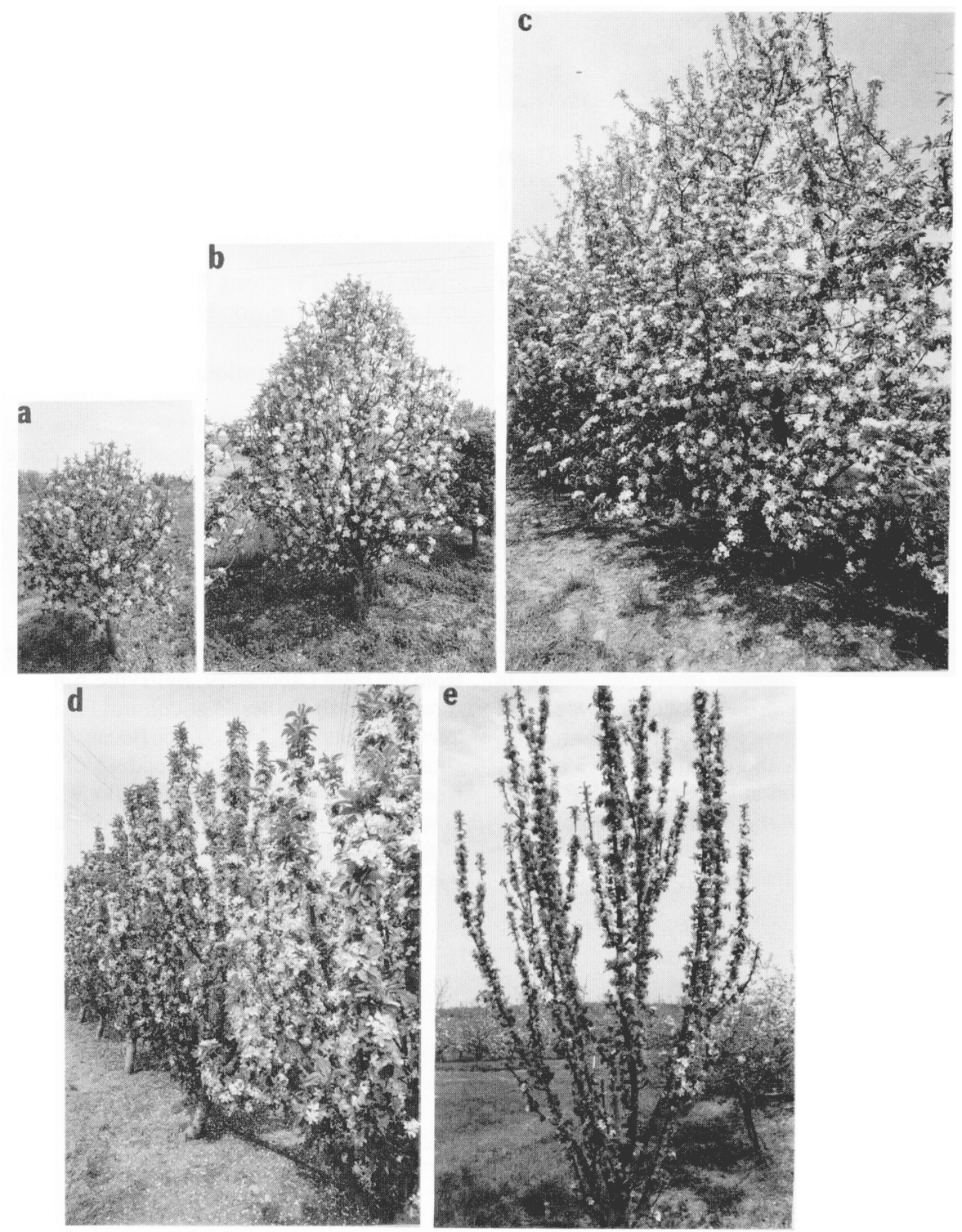

Fig.1.Dwarfappletrees,releasedasgermplasm,comparedwithstandard 'Golden Delicious' and 'McIntosh Wijcik'. Nine-year-old trees of (a) US B1, (b) US B2, (c) 'Golden Delicious', (d) US B3, and (e) 'McIntoshWijcik'.B1,B2, B3, and 'Golden Delicious' trees are self-rooted, and 'McIntosh Wijcik' is on seedling root. Pictures are proportional to the size of trees. 


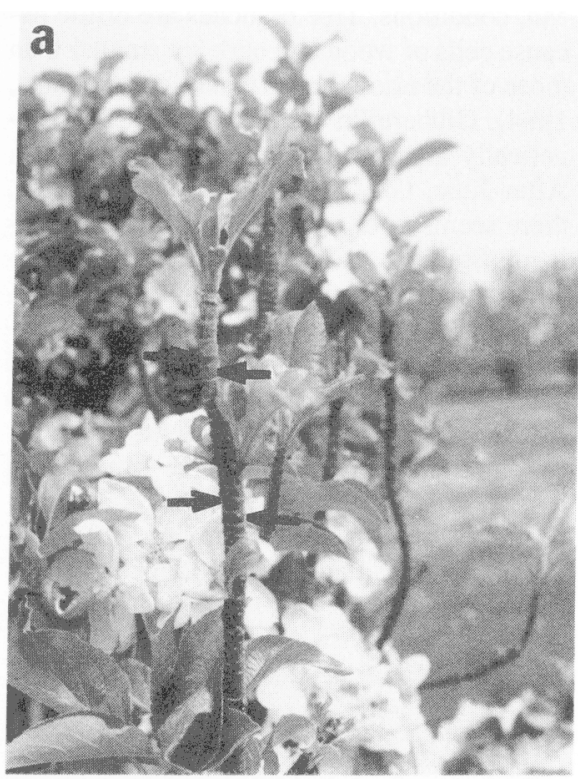

Fig.2.Internode length and amutation of US B1. (a) Leaveswere stripped to show internode length $(\approx 5 \mathrm{~mm})$. Arrows indicate location of nodes, internode is between two arrows. (b) A bud mutated to standard internode length $(\approx 30 \mathrm{rum})$ on a 4 -year-old B1 tree. Arrows indicate the mutated part of tree.

year-old trees is $\approx 2.5 \mathrm{~m}$. Internode length of the tree is somewhat longer than that of US B 1 . Although US B2 resembles US B1, leaf narrowing is absent or occurs only to a slight degree. Similar to US B1, this selection also has no summer dormancy, and the branches of the tree are brittle. The fruit is medium in size, remains green for a long time, and matures late. Fruit quality is fair to poor.

US B3 is the largest of the three introductions (Fig. id). The mean height of the 9-yearold trees is $\approx 3.5 \mathrm{~m}$. The size is similar to that of 'McIntosh Wijcik' for trees of similar age in the same plantation. US B3 is still a short internode tree. Spring internode length is slightlyshorterthan that of standard-type trees, with summer internode length being $\approx 15 \mathrm{~mm}$. Internode length shortened around 1 June at Beltsville as it did in US B 1 and US B2. The tree is upright, spurry, and has brittle branches. However, it is more branching and is not quite as spurry as 'McIntosh Wijcik' (Fig. 1e). Fruit

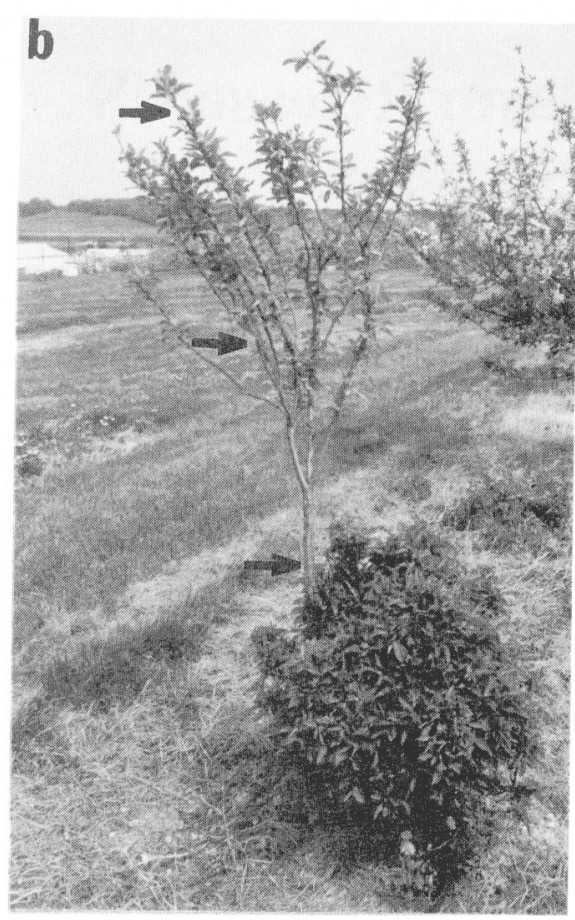

seedlings propagated by tissue culture, they are considered free of known viruses.

\section{Comments}

During the evaluation, B1, B2, and B3 trees were grafted on seedling rootstock in a separate experiment. They maintained their growth characteristics, and their size remained relatively the same or occasionally larger than the self-rooted plants. Even on seedling roots, they are usually smaller than standard trees on M.26 rootstock. Therefore, it is not necessary to graft them on size-controlling rootstock.

We do not know the chromosome numbers of these trees or the existence of possible aneuploidy. However, many trees in the 1974 progeny had similar characteristics, and this may indicate that these trees are not uniquethey are part of the natural variability of a sibcross population. B3 is a productive tree; B2 and especially B3 are less productive. We believe that when internode length is shorter than half the standard length $(30 \mathrm{~mm})$, the tree looses productivity. In contrast, the high spurriness of B3 makes it difficult to handle, and it becomes biennial easily.

\section{Literature Cited}

Faust, M. and G.L. Steffens. 1993. Correlation between internode length and tree size in apple. Acts. Hort. 349:81-84.

Grochowska,M.J., G.J. Buta, G.L. Steffens, and M Faust. 1984. Endogenous auxin and gibberellin levels in low and high vigor apple seedlings. Acts Hort. 146:125-134.

Jaumien, F. and M. Faust. 1984. Anatomical structure of shoots of Delicious and Golden Delicious apple hybrids with various growth dynamics. Acts Hort. 146:69-79.

Among the original 2000 seedlings and $\approx 500$ propagated trees of the selections, there was only one mutation (Fig. 2) found in US B1 when a bud mutated back to normal internode length (Faust and Steffens, 1993).

\section{Availability}

Limited propagation material is available from the Fruit Laboratory, Beltsville Agricultural Research Center, Agricultural Research Service, Beltsville, MD 20705. Because of the short internodes (Fig. 2a), budding of these trees may be difficult. Those who want to propagate these trees should consider grafting with woody tissues. Virus status of the trees has not been determined, but because they are

Steffens, G.L. and P. Hedden. 1992a. Comparison of growth and gibberellin concentrations in shoots from orchard-grown standard and thermosensitivedwarfapple trees. Physiol. Plant. 86:544-550.

Steffens, G.L. and P. Hedden. 1992b. Effect of temperature regimes on gibberellin levels in thermosensitive dwarf trees. Physiol. Plant. 86:539-543.

Steffens, G.L., F.W. Jacobs, and M.E. Engelhaupt 1989. Thermosensitive genetic dwarfs of apple. Physiol. Plant. 76:368-374.

Zagaja, S. and M. Faust. 1983. Population analysis of vigor and growth pattern of F2 apple seedlings with short internode parentage. J. Amer. Soc. Hort. Sci. 108:939-944. 